\title{
Magnitude, Atraso e Probabilidade de Reforço em Situações Hipotéticas de Risco ${ }^{1}$
}

\author{
Cristiano Coelho ${ }^{2}$ \\ Universidade Católica de Goiás \\ Elenice Seixas Hanna \\ Universidade de Brasília \\ João Claudio Todorov \\ Universidade Católica de Goiás e Universidade de Brasília
}

\begin{abstract}
RESUMO - O presente trabalho investigou os efeitos de quantias de dinheiro descritas como a alternativa de risco e da instrução sobre o valor subjetivo do reforço. Oito estudantes realizaram escolhas hipotéticas entre quantias de dinheiro certas ou imediatas que eram ajustadas de acordo com o comportamento dos sujeitos, e cinco quantias de magnitudes maiores ( $\mathrm{V}=$ $\mathrm{R} \$ 10,00$ a $\mathrm{R} \$ 100.000,00)$ atrasadas (uma semana a 50 anos) ou probabilísticas $(0,05$ a 0,95$)$. A instrução positiva descrevia as chances ou o atraso para receber cada quantia, enquanto a instrução negativa descrevia as chances de perder ou o tempo de espera para não perder as quantias. $\mathrm{O}$ valor subjetivo diminuiu com o aumento de $V$ provável, e aumentou com o aumento de $V$ atrasado. Os resultados individuais foram melhor descritos por uma função potência do que pela hiperbólica. A variação da instrução não produziu diferenças sistemáticas. Os resultados são evidências para uma interpretação diferente do efeito da magnitude do reforço sobre o desconto de quantias prováveis ou atrasadas.
\end{abstract}

Palavras-chave: escolha; tomada de decisão; probabilidade de reforço; magnitude de reforço; atraso de reforço.

\section{Magnitude, Delay and Probability of Reinforcer in Hypothetical Risk Situations}

\begin{abstract}
The present study investigated the effects of reinforcement magnitude of the risk alternative and different instructions on the subjective reinforcement value. Eight undergraduate students made hypothetical choices between certain immediate amounts of money that were adjusted according to the subjects' behavior, and larger amounts $(V=\mathrm{R} \$ 10,00$ to $\mathrm{R} \$ 100.000,00)$ with several delays (one week to 50 years) or probabilities $(0,05$ to 0,95$)$ associated to $V$. The positive instruction described the odds or waiting time to gain each amount of money, while the negative instruction described the odds against or the waiting time for not loosing the money. The relative subjective value decreased with increase in the probable $V$, and increased with increase in the delayed $V$. Individual results were better described by a power function than by an hyperbola. There was no systematic effect related to different instructions. The present results are evidences for a different interpretation about the effects of the reinforcement magnitude on the discount of probable or delayed amounts.
\end{abstract}

Key words: choice; decision-making; reinforcement probability; reinforcement amount; reinforcement delay.

Situações de risco podem ser definidas como aquelas nas quais o reforço depende de atraso ou probabilidade para sua liberação (Green \& Myerson, 1996; Kacelnick \& Bateson, 1996). Pode ser observado, sem necessidade de elaboração teórica, que o aumento do atraso ou a diminuição da probabilidade de um determinado evento diminui a preferência por ele, ou seja, diminui o valor que aquele evento tem para um determinado organismo. Por isso, diz-se que aos eventos atrasados ou prováveis são dados descontos (Grace, 1999).

Existe controvérsia, porém, sobre quais modelos matemáticos descrevem o desconto. Os modelos normativos de decisão postulam que os valores de alternativas prováveis ou atrasadas obedecem a regras lógico-matemáticas que incluem os valores objetivos das alternativas (Anderson,

1 Trabalho baseado na dssertação de mestrado do primeiro autor, realizada com o auxílio do CNPq, sob orientação do segundo autor. Partes do trabalho foram apresentadas na XXX Reunião Anual da Sociedade Brasileira de Psicologia, Brasília, outubro de 2000 e na XXVI Annual Convention of Association for Behavior Analysis, Washington, DC, maio de 2000.

2 Endereço: Rua 20, n.100, ap.1004 Goiânia, GO, Brasil 70020-120. E-mails: cristiano@ucg.br, hanna@unb.br, todorov@unb.br
1990; Luce, 1992). Os modelos normativos, entretanto, não prevêem diversas inconsistências de escolhas probabilísticas ou atrasadas relatadas na literatura (Allais, 1953; Kahneman \& Tversky, 1979; Lowesntein \& Prelec, 1992; Mazur, 1987; Rachlin, Raineri \& Cross, 1991; Tversky \& Kahneman, 1981).

$\mathrm{O}$ fato de escolhas inconsistentes serem observadas tanto em situações com reforçamento probabilístico quanto atrasado tem levado diversos autores a investigar se uma mesma fórmula matemática descreve as duas formas de desconto das consequências (Benzion, Rapoport \& Yagil, 1989; Christensen, Parker, Silberberg \& Hursh, 1998; Green, Myerson \& Ostaszewski, 1999; Mazur, 1997; Rachlin, Castrogiovani \& Cross, 1987; Rachlin, Logue, Gibbon \& Frankel, 1986; Rachlin \& cols., 1991; Stevenson, 1986).

Rachlin e cols. (1991) realizaram dois experimentos para investigar a hipótese de equivalência funcional entre atraso e probabilidade de reforço. No primeiro, 40 estudantes universitários escolheram entre quantias menores certas e US\$ $1.000,00$ prováveis, enquanto outros 40 escolheram entre quantias menores imediatas e US\$ 1.000,00 atrasados. Foram 
utilizadas sete probabilidades diferentes (de 0,05 a 0,95$)$ e sete atrasos (de um mês a 50 anos) para cada grupo. Rachlin e cols. utilizaram o procedimento de titulação (titration), ajustando os valores certos ou imediatos até que o sujeito indicasse uma mudança na preferência. Calculando pontos de indiferença, a partir do ponto de mudança da escolha pela quantia incerta para a quantia certa, Rachlin e cols. obtiveram, para atraso e para probabilidade, uma mesma função hiperbólica para descrever o desconto:

$$
\begin{aligned}
& \mathrm{v}=\mathrm{V} /(1+\mathrm{kD}) \\
& \mathrm{v}=\mathrm{V} /(1+\mathrm{h} \theta), \text { sendo } \theta=(1-\mathrm{p}) / \mathrm{p}
\end{aligned}
$$

onde $v$ é o valor subjetivo da quantia maior $V$ a ser recebida com atraso $D$ ou probabilidade $p ; k$ e $h$ medem a taxa de desconto produzido pelo atraso e probabilidade, respectivamente; e $\theta$ corresponde às chances contra, ou número médio de perdas em apostas a longo-prazo.

No segundo experimento, outros 40 sujeitos escolheram entre US\$1.000,00 a serem recebidos com atraso e US\$ $1.000,00$ prováveis a serem recebidos imediatamente. Os atrasos utilizados variaram de 1 mês a 100 anos, em função das taxas de desconto obtidas no Experimento 1. Para cada atraso, ajustaram-se todas as probabilidades de ganho dos US $\$ 1.000,00$, encontrando-se, assim, probabilidades equivalentes aos atrasos utilizados. Testando as funções obtidas no Experimento 1, Rachlin e cols. (1991) obtiveram uma curva paralela à prevista, com probabilidades equivalentes a atrasos menores que os previstos. Os autores atribuíram essas diferenças a questões de procedimento e a possíveis diferenças individuais, e concluíram que atraso e probabilidades subjetivos são equivalentes. Apesar disso, os autores apontaram para o fato de que a generalidade dos dados seria limitada, sendo necessário a investigação em contextos que têm influenciado diferentemente as escolhas, como com problemas, instruções ou referencial (frame) diferentes, valores e combinações entre probabilidades diversas.

Todorov e Yamane (1993) investigaram a equivalência entre atraso e probabilidade com estudantes brasileiros, em estudos semelhantes aos de Rachlin e cols. (1991). Dois experimentos foram realizados entre 1992 e 1993, quando a inflação brasileira era de 30 a $40 \%$ ao mês. No Experimento 1 , o desconto com a moeda brasileira (Cruzeiro) nas escolhas que envolviam atraso foi muito maior que o obtido por Rachlin e cols. (1991). Replicando-se o estudo (Experimento 2), agora utilizando US $\$ 1.000,00$, os dados foram semelhantes aos de Rachlin e cols. (1991). Todorov, Coelho e Hanna (1998) realizaram um terceiro experimento dois anos após o controle da inflação e obtiveram taxa de desconto com a moeda brasileira (Real), em situações com atraso, semelhante aos resultados obtidos com dólar (Todorov \& Yamane, 1993, Experimento 2e Rachlin e cols., 1991). Ostaszewski, Green e Myerson (1998) encontraram resultados semelhantes com estudantes poloneses também em duas situações distintas: uma com inflação e outra após um plano de estabilização (com inflação baixa e controlada) utilizando moedas polonesas (zloty velho, antes do plano; zloty novo, após o plano) e dólar americano, visto ser esta uma moeda-base naquela economia.

O fato de a variável inflação ter influenciado as escolhas de quantias atrasadas, mas não as escolhas de quantias prováveis, levou Todorov e cols. (1993) a concluir que atraso e probabilidade de reforçamento não são equivalentes. Além disso, a perda do valor subjetivo de quantias prováveis foi melhor descrita por uma função potência do que pela hiperbólica (Equação 2). Na equação utilizada:

$$
\mathrm{v}=\mathrm{sV}, \text { onde } \mathrm{s}=\mathrm{ap}^{\mathrm{b}}
$$

$a$ e $b$ são constantes empíricas relacionadas à perda do valor de $V$ em função da probabilidade $p$.

Um segundo achado que sustenta que a probabilidade e atraso não são equivalentes em seus efeitos sobre as escolhas deriva de estudos que manipularam também a magnitude das quantias. $\mathrm{O}$ efeito da magnitude do reforçamento sobre as escolhas com quantias atrasadas difere daquele encontrado para quantias probabilísticas: o crescimento da quantia diminui a escolha pela quantia maior probabilística, mas aumenta a escolha pela quantia maior atrasada (Christensen \& cols., 1998; Green \& cols., 1999).

Uma das grandes demonstrações das falhas dos modelos normativos de tomada de decisão foi a observação, em diversos contextos, de que pequenas modificações na forma como uma mesma alternativa é apresentada pode gerar grandes modificações sobre a decisão (Fagley \& Miller, 1987; Kahneman \& Tversky, 1979, 1984; Lowenstein, 1988; Prelec \& Lowenstein, 1991; Tversky \& Kahneman, 1981; Wang, 1996, 1996b).

Tversky e Kahneman (1981) apresentaram diferentes formulações de um mesmo problema no qual eram propostos dois planos de tratamento a pacientes hipoteticamente infectados por uma doença fatal. Um plano apresentava uma eficácia garantida, enquanto no outro a eficácia era provável, mantendo-se igual o valor médio esperado dos dois planos. Para metade dos sujeitos a instrução descrevia quantos seriam salvos com certeza no primeiro plano e, a chance de que todos fossem salvos no segundo. Para a outra metade, a instrução descrevia quantos morreriam com certeza e a chance de que todos morressem. Com a primeira instrução, $72 \%$ dos participantes escolheram a alternativa certa, enquanto com a segunda instrução $78 \%$ preferiu a alternativa provável.

Alguns autores, contudo, têm questionado até que ponto e em quais contextos esse efeito é sistemático (Schneider, 1992; Wang, 1996a, 1996b). Wang observou que, ao diminuir o tamanho do grupo hipotético de pacientes no problema vida/morte, a inversão na preferência com a mudança na instrução tende a desaparecer. Schneider (1992) aponta que o efeito de frame não ocorre em todos os contextos e depende das probabilidades utilizadas. Em seu estudo, utilizou nove cenários ou contextos diferentes, como o problema clássico de vida/morte, cura de câncer ou salvamento de plantações agrícolas e concluiu que o efeito foi mais esporádico do que uma tendência sistemática, tanto dentro de um mesmo contexto, quanto na comparação entre contextos diferentes.

Para atraso, os dados sobre o efeito de instrução são escassos. Lowenstein e Prelec (1992) apresentaram uma situação de compra de um televisor a ser pago em duas prestações, com duas versões diferentes. Uma versão apresentava apenas valores de cada prestação, na outra era apresentado um preço para o televisor e duas possibilidades de desconto. Foram mantidos constantes os valores objetivos finais das alternativas nas duas versões. Com a instrução sem desconto a maior parte dos participantes preferiu o pagamento com menor valor total, enquanto com desconto, um maior número de entrevistados afirmou preferir a alternativa que dava maior 
desconto na prestação imediata, mesmo com um pagamento total maior. Segundo Lowenstein e Prelec (1992), além de apoiarem a idéia de que as decisões são tomadas a partir de um ponto de referência, estes dados são compatíveis com o modelo hiperbólico de inversão no desconto.

$\mathrm{Na}$ Análise do Comportamento, o estudo dos efeitos de instruções tem avaliado a influência da acurácia das instruções sobre o comportamento de seguir regras (e.g., Galizio, 1979). Não há relato nesta literatura sobre o efeito de diferentes instruções que têm a mesma acurácia, mas que descrevem diferentes aspectos de uma mesma alternativa, como os trabalhos sobre o efeito de frame descritos por Tversky e Kahneman (1981) e Lowenstein e Prelec (1992).

O presente estudo teve três objetivos gerais: (1) investigar o efeito da manipulação da magnitude da alternativa de risco sobre o valor subjetivo, através da análise de dados individuais, avaliando se existe uma relação entre a taxa de desconto para probabilidade e para atraso para um mesmo participante; (2) observar se diferentes descrições de uma mesma alternativa (em termos de chance de ganho ou de perda das quantias apresentadas) geram diferenças nos valores subjetivos obtidos para um mesmo indivíduo; (3) avaliar as funções hiperbólicas e potência na descrição do desconto de quantias atrasadas e prováveis em dados individuais. Para este terceiro objetivo, avaliou-se também uma variante da função potência obtida por Todorov e cols. (1998) para situações envolvendo atraso:

$\mathrm{v}=\mathrm{rV}$, onde $\mathrm{r}=\mathrm{a}^{\prime} \mathrm{D}^{\mathrm{b}}$

sendo $a^{\prime}$ e $b^{\prime}$ constantes empíricas relacionadas à perda do valor da quantia $V$ em função do atraso $D$. Estudos anteriores avaliaram os efeitos de quantia de dinheiro em dados de grupo (Christensen \& cols. 1998; Green \& cols., 1999; Kirby \& Maracovic, 1996), ou individuais apenas para atraso ( Kirby, 1997; Myerson \& Green, 1995). Enquanto Green e cols. (1999) apontam que o aumento do desconto com probabilidade e a diminuição com atraso em função do aumento da quantia estão relacionados à questão do autocontrole (com os efeitos opostos tornando as escolhas mais autocontroladas à medida que aumenta o valor), o presente trabalho visa investigar se há uma tendência individual de comportamento impulsivo.

\section{Método}

\section{Participantes}

Oito estudantes de graduação matriculados em disciplinas introdutórias do curso de Psicologia da Universidade de Brasília, sem experiência prévia em estudos relacionados a este, participaram voluntariamente do estudo. Cinco eram do sexo feminino e três do sexo masculino, com idades entre 19 e 23 anos. Todos receberam certificados de participação a serem trocados por pontos em disciplinas, de acordo com o número de horas.

\section{Material}

Conjunto de cartões contendo os valores, probabilidades ou atrasos utilizados e uma prancheta com locais determinados para a apresentação de quantias menores certas ou ime- diatas, dos valores maiores e das probabilidades ou atrasos associados a estes valores.

\section{Procedimento}

A tarefa consistiu em situações hipotéticas de escolha entre uma quantia pequena $(v)$ certa ou imediata e outra maior $(V)$ atrasada ou provável. Foram utilizados 27 valores menores para cada $V$, variando de $0,01 \mathrm{~V}$ a $0,99 \mathrm{~V}$. Os atrasos utilizados foram de 50 anos, dez anos, cinco anos, um ano, seis meses, um mês e uma semana, e as probabilidades (expressas em porcentagens) 5\%, 10\%, 30\%, 50\%, 70\%, $90 \%$ e $95 \%$. Para cada atraso e cada probabilidade, os valores menores imediatos ou certos eram aumentados, em escolhas independentes, até que o participante passasse a escolher a quantia imediata ou certa, e mantivesse sua preferência por esta alternativa por quatro escolhas consecutivas. Enquanto $v$ era aumentado, o atraso associado a $V$ permanecia fixo. Após ser atingido o critério de mudança com aquele atraso, trocava-se o cartão de atraso e o procedimento era reiniciado com o valor imediato $0,01 \mathrm{~V}$. O mesmo procedimento foi realizado com todos os atrasos, de 50 anos a uma semana. Após o último atraso, iniciavam-se as escolhas com probabilidade, utilizando-se o mesmo procedimento, de 5\% a $95 \%$.

Este procedimento foi repetido com cinco magnitudes de $V(\mathrm{R} \$ 10,00, \mathrm{R} \$ 100,00, \mathrm{R} \$ 1.000,00, \mathrm{R} \$ 10.000,00$, e $\mathrm{R} \$ 100.000,00)$ em diferentes sessões. Para cinco participantes $V$ diminuiu entre sessões, e aumentou para os outros três.

Além das instruções gerais, o participante recebia uma instrução específica com frame positivo ou negativo. Na que foi denominada de frame positivo, a instrução descrevia os valores menores a serem recebidos e as probabilidades ou atraso para recebimento de $V$.

Em cada escolha o experimentador perguntava ao participante: "o que você prefere, esperar $D$ tempo (10 anos) para ganhar $V$ (cem reais) ou ganhar $v$ (um real) agora?" para problemas que envolviam atraso. Para os problemas com probabilidade de reforço perguntava-se: "o que você prefere, tentar ganhar $V$ ( cem reais) tendo $p$ de chance $(90 \%)$ ou receber $v$ (um real) com certeza?".

A instrução com referencial negativo descrevia a parte de $V$ a ser perdida na alternativa certa ou imediata, a chance de não receber $V$ ou o tempo de espera para não perder $V$. Assim nos problemas que envolviam atraso perguntava-se: “o que você prefere, esperar $D$ tempo ( 10 anos) para não perder nada de $V$ ( cem reais) ou receber agora, perdendo $V$-v (noventa reais) de $V$ (cem reais)?". Para situações com probabilidade o enunciado era: "o que você prefere, arriscar em $V$ (cem reais), tendo 100-p (10\%) de chance de perdê-lo, ou receber com certeza, perdendo $V-v$ (noventa e nove reais) de $V$ (cem reais)?".

Para cada referencial, os participantes foram testados com todos os valores maiores, para posteriormente realizar as escolhas com o outro referencial. Metade dos participantes começou com referencial positivo e a outra com o negativo.

\section{Resultados}

A principal medida utilizada para análise foi o ponto de indiferença entre magnitudes certas ou imediatas e magni- 
Probabilidade



Figura 1. Razão de indiferença como função da magnitude da quantia provável com instrução positiva e negativa para os participantes AM, CM, LT e RR. Curvas diferentes apresentam os resultados de cada probabilidade utilizada.

tudes prováveis ou atrasadas, calculado a partir da média entre o maior $v$ escolhido e o menor $v$ com o qual os participantes preferiram a quantia atrasada ou a provável. Este cálculo foi realizado para todos os $V$ utilizados, com todas as probabilidades e atrasos separadamente, com as instruções positiva e negativa. Esses pontos foram transformados em razões de indiferença, consistindo da razão entre os pontos de indiferença obtidos e o $V$ utilizado $(v / V)$. Cada conjunto de sete pontos obtidos para cada $V$ gerou curvas denominadas curvas de indiferença. 
A Figura 1 apresenta as curvas de indiferença obtidas para cada probabilidade, como função da magnitude de $V$, com instrução, para participantes cujos resultados são representativos da amostra. Ao comparar as diferentes razões obtidas para um mesmo $V$, pode-se observar aumentos nas razões de indiferença com o aumento na probabilidade de ganho de $V$. Analisando-se os dados obtidos para uma mesma probabilidade, observa-se que diminuíram as razões de indiferença, com o aumento da quantia provável $(V)$. Excetuam-se a esta tendência os dados obtidos por AC (p. 0,05 e 0,1, instruções positivas, todas as probabilidades com instruções negativas), AM (p. 0,95 e instrução negativa), MF e RR (p. 0,95, 0,9 e 0,7 , com instruções positivas, e os dados obtidos com instruções negativas).

Para a maior parte dos resultados, a razão de indiferença permaneceu abaixo do valor médio esperado (modelo normativo), que consiste na multiplicação da probabilidade de ganho pelo valor a ser recebido. Acima deste valor permaneceram apenas as razões obtidas para $\mathrm{R} \$ 10,00$ e $\mathrm{R} \$ 100,00$, e principalmente para as probabilidades mais baixas, para os participantes MF, CM e RF. Os participantes AC e RR obtiveram razões de indiferença mais altas que os valores médios para as probabilidades de 0,05 a 0,30 com todos os $V$. Os resultados com instrução negativa para LT apresentam razões de indiferença mais altas que os valores médios para $\mathrm{R} \$ 10.000,00$ e $\mathrm{R} \$ 100.000,00$.

As razões de indiferença obtidas através da manipulação do atraso do recebimento de $V$ são apresentadas na Figura 2, para os mesmos quatro participantes. Ao comparar as diferentes razões obtidas para um mesmo $V$, observa-se a diminuição da razão de indiferença com o aumento no atraso para recebimento de $V$. De uma forma geral, a perda do valor de $V$ em função do aumento do atraso gerou razões de indiferença iguais ao menor valor possível (correspondendo a $0,005 \mathrm{~V}$ ) antes dos 50 anos na maioria dos casos (exceto para os sujeitos LT e RR), gerando valores mais próximos entre todos os $V$ para os atrasos maiores. A espera com $\mathrm{R} \$ 10,00$ e $\mathrm{R} \$ 100,00$ foi ainda menor, obtendo-se valores subjetivos iguais a $0,05 \mathrm{~V}$ com atrasos ainda menores que para as demais quantias $V$.

Outro resultado sistemático foi o aumento da razão de indiferença com o aumento de $V$ para a maior parte dos atrasos. Para a metade dos participantes (AC, AM, LT e RR), as razões de indiferença aumentaram até a quantia de $\mathrm{R} \$ 100.000,00$. Para a outra metade, o aumento foi observado até $\mathrm{R} \$ 10.000,00$, voltando a diminuir com $\mathrm{R} \$ 100.000,00$. As curvas de indiferença obtidas com $\mathrm{R} \$ 10,00$ e $\mathrm{R} \$ 100,00$ ficaram próximas para AM, com instrução negativa e para MF, com instrução positiva. Observaram-se também, curvas próximas entre $\mathrm{R} \$ 100,00 \mathrm{e}$ $\mathrm{R} \$ 1.000,00$ para AM e para RF, com instrução positiva, para a maior parte dos atrasos investigados.

Foram também realizadas comparações entre as diferentes curvas de indiferença obtidas com as duas instruções. Duas questões foram avaliadas: primeiro, se haveria diferença entre as razões de indiferença obtidas com cada instrução para um mesmo $V$; segundo, se existiria alguma relação entre essas diferenças em função da manipulação de $V$. As curvas de indiferença obtidas com as duas instruções foram semelhantes para todos os $V$ utilizados. Para probabilidade, observou-se diferenças para $\mathrm{R} \$ 100.000,00, \mathrm{R} \$ 1.000,00$ e $\mathrm{R} \$ 10,00$, e para sujeitos específicos ( $\mathrm{AC}, \mathrm{LT}$ e MF), não sendo observada, em geral, uma variação sistemática das razões de indiferença. Para atraso, as semelhanças entre os valores subjetivos com ambas as instruções foram ainda maiores.

A partir das curvas de indiferença obtidas para cada sujeito, avaliou-se o ajuste da função hiperbólica (através de transformações das Equações 1 e 2) e da função potência (com transformações das Equações 3 e 4) para atraso e para probabilidade. Para esta análise realizou-se cálculo de regressão linear, utilizando o método dos mínimos quadrados, através da inversão da função hiperbólica e transformação logarítmica da função potência.

Os coeficientes de determinação de ambas as funções para cada valor $\mathrm{V}$ e instrução foram maiores para probabilidade do que para atraso, para a maior parte das regressões realizadas. As médias dos ajustes da função hipérbole foram $0,75(D P$ $=0,19)$ e $0,72(D P=0,19)$, nas escolhas com probabilidade, e 0,55 $(D P=0,29)$ e $0,51(D P=0,27)$, nas escolhas com atraso, com instrução positiva e negativa, respectivamente. A função potência apresentou ajustes médios de $0,87(D P=$ $0,09)$ e $0,86(D P=0,14)$ para probabilidade, e $0,79(D P=$ $0,11)$ e $0,77(D P=0,11)$ para atraso.

Nas escolhas com probabilidade, o ajuste da função potência foi significativo com todos os valores, exceto para AC, com instrução negativa e $V$ igual a $\mathrm{R} \$ 100.000,00$. Para as escolhas com atraso, o ajuste da função potência não foi significativo apenas para LT, com $\mathrm{R} \$ 10.000,00$, com as duas instruções. A função hipérbole apresentou um número menor de ajustes significativos, principalmente nas escolhas com atraso. Para probabilidade, de um total de 43 análises realizadas com instrução positiva, em $30 \%$ a função hipérbole apresentou ajustes não significativos. Com instrução negativa, os ajustes não foram significativos em $28 \%$ das 44 análises realizadas. Esses ajustes com baixa significância foram obtidos principalmente para escolhas do sujeito AM, para o qual nenhuma análise foi significativa.

Para atraso, o ajuste da hipérbole foi significativo em 56\% das regressões. Para a função hipérbole, a única quantia em que se obteve ajustes significativos para todos os participantes foi $\mathrm{R} \$ 100.000,00$. Além disso, para $\mathrm{R} \$ 10,00$ e $\mathrm{R} \$ 100,00$, o $\mathrm{R}^{2}$ foi significativo apenas para $\mathrm{RR}$.

Essas regressões permitiram que se obtivesse os valores das constantes individuais de desconto da função hipérbole e da função potência. A Figura 3 apresenta os valores das constantes $k$ (para atraso, gráficos à direita) e $h$ (para probabilidade, gráficos à esquerda) das Equações 3 e 4, respectivamente, para cada valor a partir das análises nas quais o ajuste desta função foi significativo. Cada linha apresenta os resultados de um sujeito, para todos os valores de $V$. A taxa de desconto relativa à magnitude $(h)$ aumentou com o aumento em $V$, com instruções positivas para CM, LT, MF e RR, e com instruções negativas para AG, LT e RF. Contudo, com instruções negativas, esse aumento não foi observado com todos os valores de $V$. Para os demais sujeitos não houve variação sistemática de $h$ com o aumento de $V$ ou não foi possível a análise, dado o pequeno número de pontos. Para as escolhas com atraso, a taxa de desconto $(k)$ para os sujeitos CM, LT e RR diminuiu com aumento no valor de $V$, com as duas instruções. Para os demais participantes não foi possível realizar esta comparação em função da pequena quantidade de pontos. 
Atraso

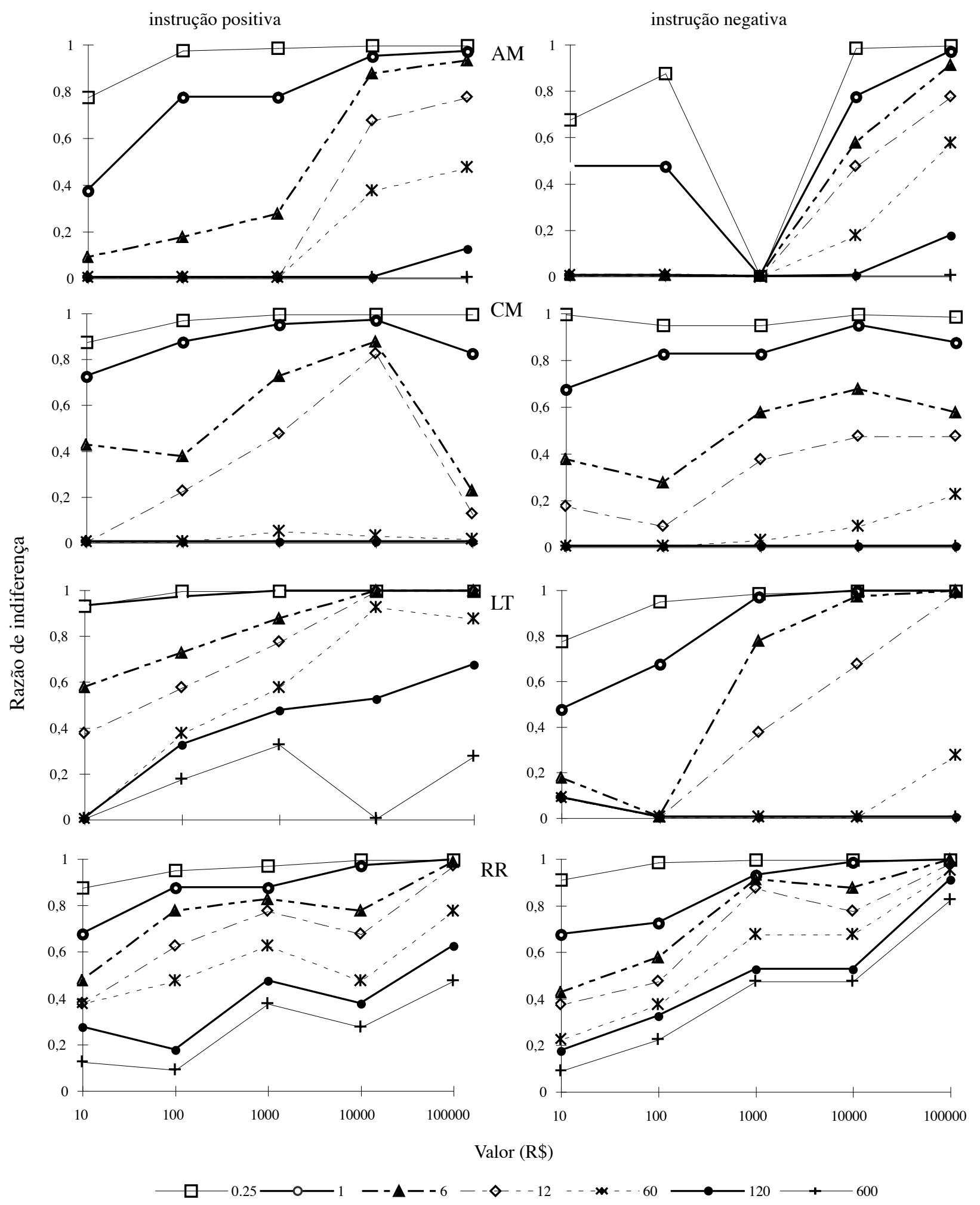

Figura 2. Razão de indiferença como função da magnitude da quantia atrasada com instrução positiva e negativa para os participantes AM, CM, LT e RR. Curvas diferentes apresentam os resultados de cada atraso (em meses) utilizado.

A Figura 4 apresenta os parâmetros $b$ e $b^{\prime}$ (dois primeiros pares de gráficos) e $\log (a)$ e $\log \left(a^{\prime}\right)$ (dois últimos pares de gráficos) da função potência (Equações 3 e 4), utilizando-se apenas as constantes obtidas em regressões com ajuste sig- nificativo. O cálculo das constantes foi realizado através da transformação em sua forma logarítmica, na qual se obtém uma função linear com inclinação igual a $b$ ou $b$ ' e intercepto igual a $\log (a) \log \left(a^{\prime}\right)$. 


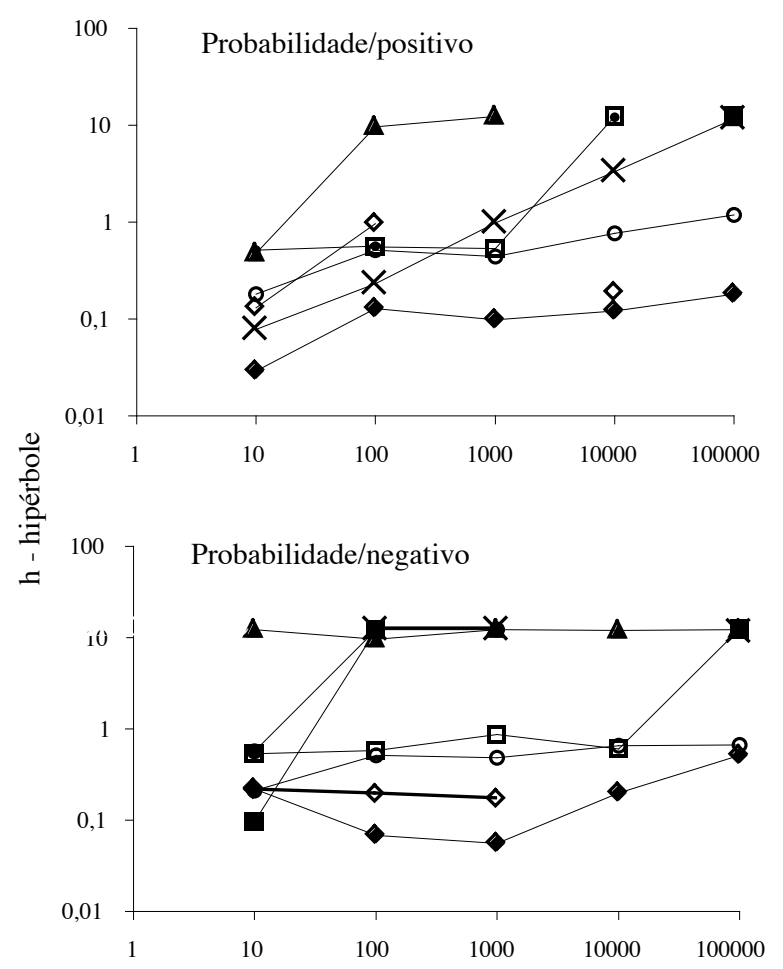

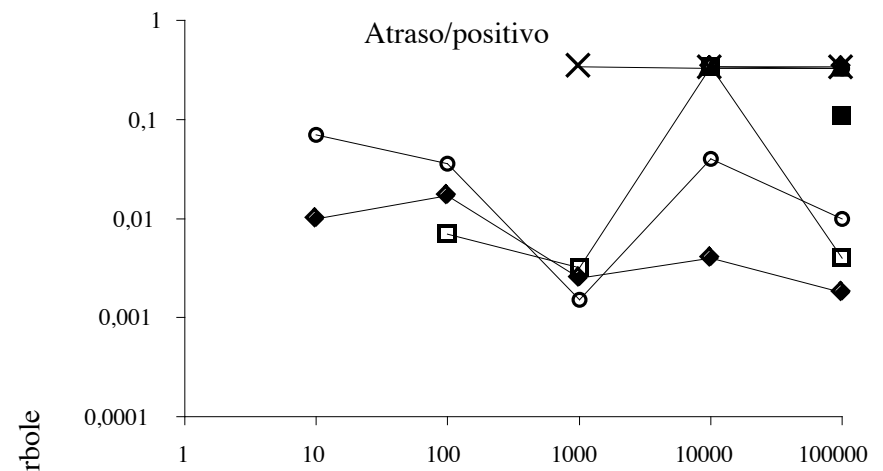

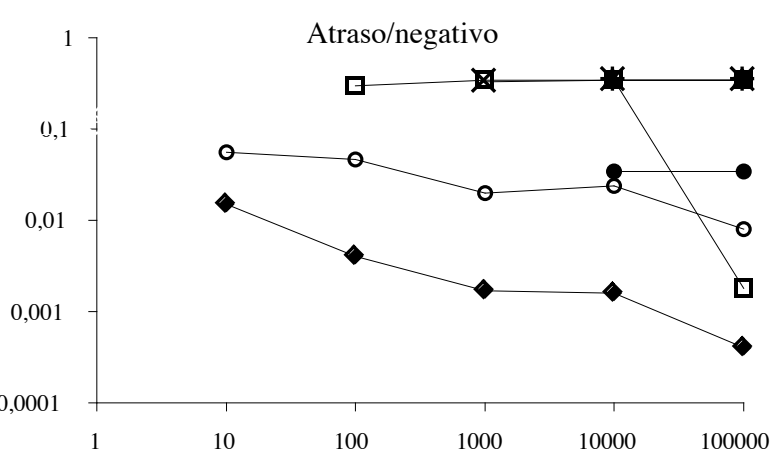

Valor $(\mathrm{R} \$)$

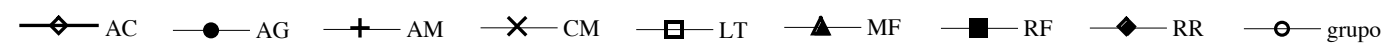

Figura 3. Valores das constantes da função hipérbole para probabilidade ( $h$, gráficos à esquerda) e atraso $(k$, gráficos à direita), obtidas para todos os participantes e para os dados de grupo com todas as magnitudes de $V$, para cada instrução. São apresentadas apenas constantes obtidas em ajustes significativos.

Para probabilidade não foi observada, na maior parte dos casos, uma tendência ascendente ou descendente para o parâmetro $b$, exceto para CM e MF, para os quais $b$ aumentou com o aumento de $V$, nas escolhas com instrução positiva. Para atraso, os valores de $b$ obtidos por LT, RR e para o dado de grupo aumentaram com o aumento de $V$. Observou-se uma tendência descendente no valor de $\log (a)$, e ascendente no valor de $\log \left(a^{\prime}\right)$, em função do aumento de $V$. Para probabilidade, observou-se para alguns sujeitos, pontos que fugiram a esta tendência para $\mathrm{R} \$ 100,00$ e $\mathrm{R} \$ 10.000,00$, com instrução positiva (AC, AG e LT) e para os valores $\mathrm{R} \$ 100,00$ (RF) e $\mathrm{R} \$ 1.000,00(\mathrm{CM})$ com instrução negativa. Para atraso com instrução positiva, o valor de $\log \left(a^{\prime}\right)$ obtido com $\mathrm{R} \$ 10.000,00$ também desviou da tendência citada para os sujeitos AG, CM e MF.

\section{Discussão}

O presente trabalho investigou: (1) a influência da magnitude da alternativa de risco sobre a escolha em situações que envolvem atraso ou probabilidade do reforço; (2) se a instrução utilizada com o procedimento de ajustamento da quantia menor certa ou imediata influencia os valores subjetivos das alternativas; e (3) se as funções de valor subjetivo que têm descrito dados de grupo, descrevem o desconto para dados individuais.

Os resultados mostraram que a razão de indiferença aumenta com aumentos da quantia a ser recebida com atraso, e diminui com o aumento do valor provável, confirmando e estendendo os resultados de Christensen e cols. (1998), Green e cols. (1999) e Kirby (1997).

A manipulação da instrução, em geral, não produziu efeitos sistemáticos sobre os valores subjetivos do reforço para as diferentes magnitudes. Esses resultados apóiam Schneider (1992), que afirma que o efeito de instrução é mais exceção do que uma regra geral.

Com relação à análise da função que descreve o valor subjetivo em situações de risco, encontrou-se que a função potência proposta por Todorov e cols. (1998), com duas medidas relacionadas à perda do valor do reforço $(a \mathrm{e} b$, Equação 4), descreveu melhor os resultados do presente estudo do que os modelos hiperbólicos utilizados por Mazur (1987) e Rachlin e cols. (1991). Esses resultados estendem a descrição do valor do reforço proposta de Todorov \& Yamane (1993) e Todorov e cols. (1998) para situações com atraso.

A não ocorrência de diferenças sistemáticas com a manipulação da instrução sugere que as variações observadas devem-se a maior complexidade da instrução negativa, que permite o estabelecimento de controle comportamental diferenciado em participantes com histórias diferentes. Esses resultados replicam, ao nível individual, os dados obtidos por Hershey e Schoemaker (1980, conforme citado por Schneider, 1992), mas contrastam com os relatados por Tversky e Kahneman (1981). A ausência de efeito da instrução pode estar também relacionada ao grande número de escolhas que cada participante realizou, configurando-se como um treino extensivo na tarefa e diminuindo as diferenças entre os resultados com as duas instruções. 

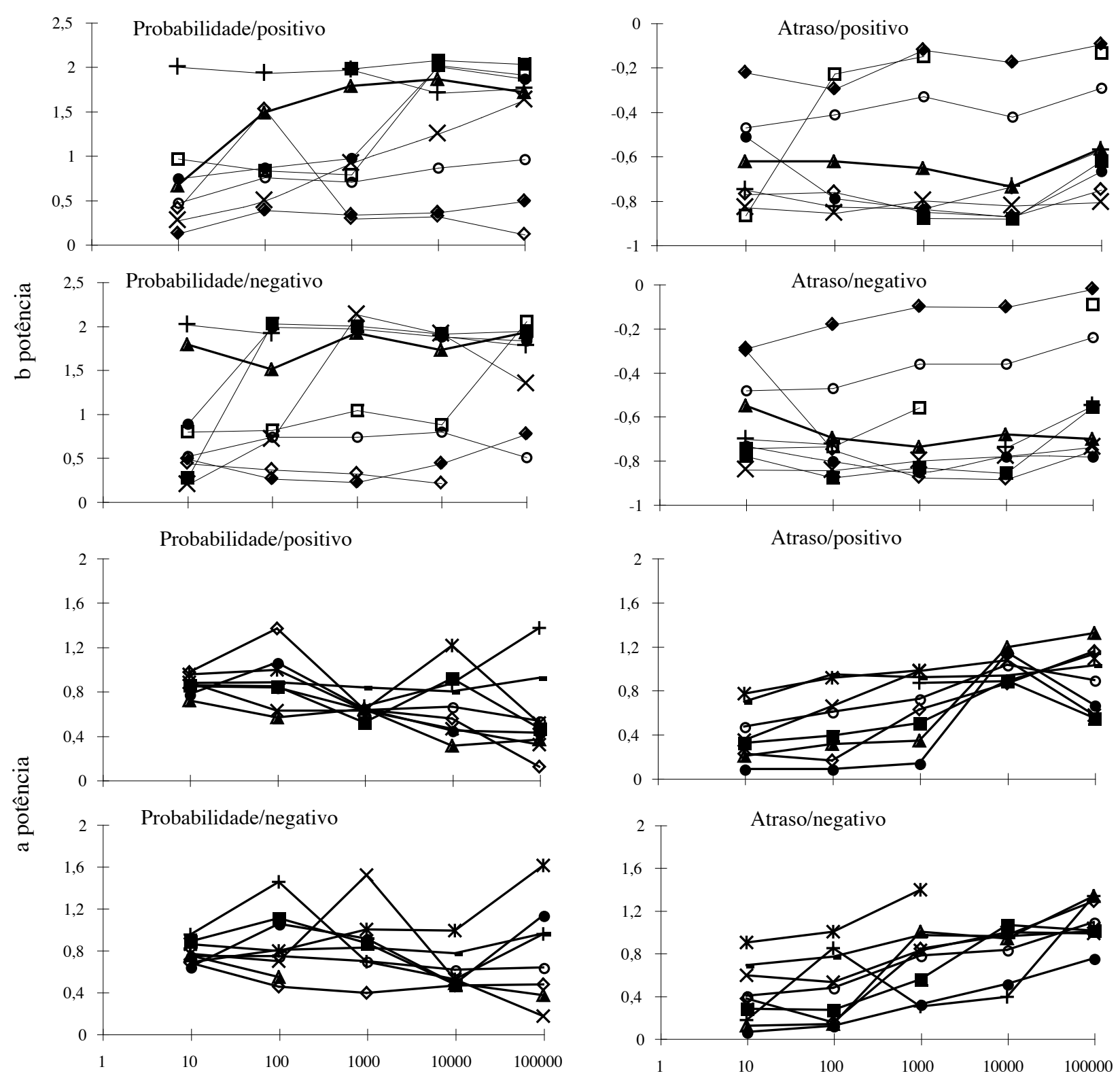

Valor (R\$)

Figura 4. Constantes da função potência (b, b' - parte superior; a, a' - parte inferior) para probabilidade (gráficos à esquerda) e atraso (gráficos à direita), calculadas para cada participante e para os dados de grupo. São apresentadas apenas constantes obtidas em ajustes significativos.

A razão de indiferença aumentou com aumentos em $V$ para escolhas com atraso, e diminuiu com aumentos na quantia a ser recebida dependendo de uma probabilidade. Esses achados replicam os relatados na literatura de que o valor $V$ tem influências opostas sobre o desconto com atraso e probabilidade (Green e cols., 1999). Para alguns sujeitos, os valores de indiferença para $\mathrm{R} \$ 100,00, \mathrm{R} \$ 1.000,00 \mathrm{e}$ $\mathrm{R} \$ 10.000,00$ foram próximos para a maioria dos atrasos e probabilidades. Contudo, foi verificado um aumento não contínuo do desconto em função do aumento da magnitude da alternativa provável. Esse resultado difere do relatado na literatura (Green e cols., 1999) com análises de grupo.
Para atraso, os valores subjetivos relativos mais altos para $\mathrm{R} \$ 10.000,00$ do que para $\mathrm{R} \$ 100.000,00$ para alguns participantes sugerem que a influência da manipulação da magnitude sobre as escolhas com atraso segue uma curva bitônica, conforme encontrado por Green e cols. (1999). Isto é, o desconto de quantias atrasadas pode diminuir até um determinado valor, mas volta a aumentar para valores ainda maiores. Mesmo assim, fazem-se necessários estudos para avaliar os efeitos de quantias maiores que $\mathrm{R} \$ 100.000,00$.

As funções potência (Equações $3 \mathrm{e} 4$ ) descreveram melhor que as hipérboles propostas por Mazur (1987) e Rachlin e cols. (1991) os dados para a maioria dos sujeitos. Deve-se 
observar, porém, que nas escolhas com atraso, em alguns casos, a função hipérbole se ajustou melhor que a potência para $\mathrm{R} \$ 100.000,00$. Observaram-se também, casos nos quais ambas as funções apresentaram baixos valores de $\mathrm{R}^{2}$, sugerindo a possibilidade de funções diferentes de desconto. Além disso, variáveis históricas não controladas podem ter contribuído para alguns ajustes baixos para ambas as funções. Por exemplo, AC, com instrução positiva com $\mathrm{R} \$ 100.000,00$, parou em um mesmo valor, independente da probabilidade. Durante a sessão, sempre que chegava a este valor ela relatava "a partir daqui eu pego o imediato; já dá para comprar um carro". Em estudos que utilizam análises de grupo, as diferenças individuais geradas por essas formas de controle são minimizadas. Esta pode ser uma das possíveis causas de diferenças entre resultados de grupo relatados na literatura e os resultados individuais apresentados no presente estudo.

$\mathrm{O}$ teste da função potência para escolhas com atraso ainda não havia sido realizado em estudos anteriores. $\mathrm{O}$ presente trabalho mostrou que é uma proposta viável de análise para estas situações, principalmente na descrição de escolhas com baixos valores de $V$, descrevendo adequadamente os resultados. Para probabilidade, os resultados do presente estudo replicaram os obtidos por Todorov e cols. (1998), mostrando uma maior força descritiva da função potência. Não há nenhum outro relato do teste dessa função para escolhas em situação de risco na literatura e, portanto, reanálises de estudos anteriores poderiam ser feitas a fim de se investigar se os resultados podem ser melhor descritos por esta função.

Green e cols. (1999) relataram que as diferenças encontradas nas curvas de desconto para diferentes valores, com atraso e probabilidade, devem-se a variações no valor dos parâmetros $k$ e $h$, respectivamente. Enquanto $k$ aumentaria com a diminuição de $V$, o parâmetro $h$ diminuiria. Apesar do presente estudo ter encontrado um efeito semelhante ao de Green e cols. (1999) da manipulação do valor sobre as razões de indiferença, a análise em termos de variações nos parâmetros $k$ e $h$ é dificultada pelo fato de que as funções hipérboles para probabilidade e para atraso não descreveram adequadamente os dados, com vários valores de $\mathrm{R}^{2}$ muito baixos. Mesmo assim, para as análises significativas, observou-se um aumento no valor de $h$ para quatro sujeitos com instruções positivas e para três sujeitos com instruções negativas. Nas escolhas com atraso com instrução positiva, observaram-se três casos em que o valor de $k$ aumentou, e outros três sem tendência ascendente ou decrescente. Em outros casos, razões diferentes deveram-se a variações no ponto em que as curvas cruzaram o eixo Y com a manipulação de $V$, variações estas não previstas pela função hiperbólica.

Tomando-se os resultados descritos pela função potência, duas relações podem ser derivadas, a partir da transformação logarítmica da função potência. A primeira derivada da análise do ponto em que a razão de indiferença intercepta o eixo $Y$, que corresponde a $\log (a)$ para probabilidade e $\log \left(a^{\prime}\right)$, para atraso. A segunda corresponde à perda do valor entre as diferentes manipulações de atraso ou de probabilidade, ou seja, a inclinação das curvas de valor subjetivo obtidas com cada um dos valores, que corresponde a $b$ ou $b^{\prime}$. Enquanto as funções hiperbólicas de Mazur (1987) e Rachlin e cols. (1991) prevêem que apenas a aceleração na perda do valor da razão de indiferença é afetada pela manipulação da probabilidade ou do atraso, a função potência prevê diferenças nos valores subjetivos também quando os interceptos são diferentes, mas não o grau de perda, definido por $b$ ou de $b$ ', para todas as probabilidades ou atrasos. A tendência observada mostra variações não sistemáticas de $b$ e de $b$ ' na maior parte dos casos, mas uma tendência de diminuição no intercepto $(\log (a))$ para as escolhas com probabilidade, e um aumento no $\log \left(a^{\prime}\right)$ para escolhas envolvendo atraso, em função do aumento do valor maior. Em alguns casos, porém, os valores do $\log (a)$ ou $\log \left(a^{\prime}\right)$ para $\mathrm{R} \$ 100,00$ e $\mathrm{R} \$ 10.000,00$ foram maiores que os obtidos com os demais valores, casos em que estes valores se desviaram da tendência geral, ou que as diferenças nos valores subjetivos relativos decorreram da interação entre as duas medidas: a inclinação e o intercepto. A título de exemplificação, em alguns casos, observaram-se razões de indiferença mais altas para $R \$ 10.000,00$ com baixas probabilidades, comparadas às obtidas com $\mathrm{R} \$ 100.000,00$, denotando um intercepto maior para aquele valor.

Os presentes resultados são evidências para uma nova interpretação para a influência de $V$ sobre o desconto de quantias prováveis e atrasadas. $\mathrm{O}$ aumento da quantia atrasada diminui o viés (aumentando o intercepto), enquanto o aumento da quantia provável aumenta o viés (com a diminuição do intercepto) em favor da magnitude certa e imediata, respectivamente. Os resultados apontam ainda para a possibilidade de que a impulsividade esteja relacionada não apenas à aceleração da perda do valor do reforço, mas principalmente ao viés em favor da alternativa imediata. Esta análise também pode abarcar a mudança na preferência em função do aumento do atraso, ou da diminuição da probabilidade previstas pela função hiperbólica e pela Lei da Igualação (Davison, 1988).

Com essa nova interpretação, novas possibilidades podem ser levantadas na discussão sobre a influência do valor absoluto sobre o desconto. Ao se observar que não há variações sistemáticas na inclinação, e sim no intercepto, esses dados são semelhantes aos obtidos sobre efeitos de magnitude com pombos: há influência sobre o viés, e não sobre a sensibilidade do comportamento a variações nos valores absolutos da magnitude (Fantino \& Davison, 1983).

A generalidade dos presentes resultados é, contudo, restrita. Outras formas de investigação dos efeitos da manipulação da instrução devem ser realizadas. Além disso, deve-se investigar a aplicabilidade dessas equações utilizando-se outros procedimentos - notadamente procedimentos operantes, nos quais a recompensa esteja disponível - e outros organismos. Outra questão refere-se ao fato de terem sido utilizadas quantias hipotéticas. Parece possível supor que a utilização de quantias hipotéticas pode diminuir as restrições à escolha realizada e produzir, portanto, resultados diferentes quando outras fontes de controle comportamental estiverem presentes.

\section{Referências}

Allais, M. (1953). Le comportement de l'homme rationnel devant le risque: Critique des postulats et axiomes de l'école américaine. Econometrica, 21, 503-546.

Anderson, J.R. (1990). Cognitive Psychology. New York: Freeman. 
Benzion, U., Rapoport, A. \& Yagil, J. (1989). Discount rates inferred from decisions: an experimental study. Management Sciences, 35, 270-284.

Christensen, J., Parker, S., Silberberg, A. \& Hursh, S. (1998). Trade-offs in choice between risk and delay depend on monetary amounts. Journal of the Experimental Analysis of Behavior, 69, 123-139.

Davison, M. (1988). Delay of reinforcers in a concurrent-chain schedules: an extension of the hyperbolic-decay model. Journal of The Experimental Analysis of Behavior, 50, 219-236.

Fagley, N.S. \& Miller, P.M. (1987). The effects of decision framing on choice of risk vs certain options. Organizational Behavior and Human Decision Processes, 39, 264-277.

Fantino, E. \& Davison, M. (1983). Choice: Some quantitative relations. Journal of the Experimental Analysis of Behavior, 40, 1-13.

Galizio, M. (1979). Contingency-shaped and rule governed behavior: instructional control of human loss avoidance. Journal of the Experimental Analysis of Behavior, 31, 53-70.

Grace, R. (1999). The matching law and amount-dependent functions as account for choice in human and pigeon. Journal of the Experimental Analysis of Behavior, 70, 1-19.

Green, L. \& Myerson, J. (1996). Exponential versus hyperbolic discounting of delayed outcomes: risk and waiting time. American Zoologist, 36, 496-505.

Green, L., Myerson, J. \& Ostaszewski (1999). Amount of reward has opposite effects on the discounting of delayed and probabilistic outcomes. Journal of Experimental Psychology: Learning, Memory and Cognition, 25, 418-427.

Kacelnick, A. \& Bateson, M. (1996). Risky theories - the effects of variance on foraging decisions. American Zoologist, 36, 402-434.

Kahneman, D. \& Tversky, A. (1979). Prospect theory: an analysis of decision under risk. Econometrica, 47, 263-291.

Kahneman, D. \& Tversky, A. (1984). Choice, values, and frames. American Psychologist, 39, 341-350.

Kirby, K. (1997). Bidding on the future: evidence against normative discounting of delayed rewards. Journal of Experimental Psychology: General, 126, 54-70.

Kirby, K. \& Marakovic (1996). Delay-discounting probabilistic rewards: rates decrease as amounts increase. Psychonomic Bulletin \& Review, 3, 100-104.

Lowenstein, G. (1988). Frames of mind in intertemporal choice. Management Sciences, 34, 200-214.

Lowenstein, G. \& Prelec, D. (1992). Anomalies in Intertemporal choice: Evidence and interpretation. Em G. Lowenstein \& J. Elster (Orgs.), Choice over time (pp. 119-146). New York: Russel Sage Foundation.

Luce, R. D. (1992). Where does subjective expected utility fail descriptively? Journal of Risk and Uncertaint, 5, 5-27.

Mazur, J. E. (1987). An adjusting procedure for studying delayed reinforcement. Em M.L. Commons, J.E. Mazur, J.A. Nevin \& H. Rachlin (Orgs.). Quantitative Analysis of Behavior: Vol 5. The effect of delay and of intervening events on reinforcement value (pp. 55-73) Hillsdale, NJ: Erlbaum.

Mazur, J.E. (1997). Choice, delay, probability and conditioned reinforcement. Animal Learning and Behavior, 25, 131-147.

Myerson, J. \& Green, L. (1995). Discounting of delayed rewards: models of individual choice. Journal of the Experimental Analysis of Behavior, 64, 263-276.
Ostaszewski, P., Green, L. \& Myerson, J. (1998). Effects of inflation on the subjective value of delayed and probabilistic rewards. Psychonomic Bulletin and Review, 5, 233-244.

Prelec, D. \& Lowenstein, G. (1991). Decision making over time and under uncertainty: a common approach. Management Sciences, 37, 770-786.

Rachlin, H., Castrogiovani, A. \& Cross, D. (1987). Probability and delay in commitment. Journal of the Experimental Analysis of Behavior, 48, 347-353.

Rachlin, H., Logue, A.W., Gibbon, J. \& Frankel, M. (1986). Cognition and behavior in studies of choice. Psychological Review, 93, 33-45.

Rachlin, H., Raineri, A., \& Cross, D. (1991). Subjective probability and delay. Journal of the_Experimental Analysis of Behavior, 55, 233-244.

Schneider, S. (1992). Framing and conflict: Aspiration level contingence, the status quo and current theories of risky choice. Journal of Experimental Psychology: Learning, Memory and Cognition, 18, 1040-1057.

Stevenson, M.K. (1986). A discounting model for decisions with positive and negative outcomes. Journal of Experimental Psychology: General, 115, 131-154.

Todorov, J.C., Coelho, C. \& Hanna, E. S. (1998, maio). Subjective value: on the equivalence between probability and delay. Trabalho apresentado na XXIV Convenção Anual da Association for Behavior Analysism, Orlando, USA.

Todorov, J.C. \& Yamane, A.M. (1993). Subjetividade, probabilidade e demora: decisões em situações de risco. Manuscrito não publicado, Universidade de Brasília.

Tversky, A. \& Kahneman, D. (1981). The framing of decisions and the psychology of choice. Science, 211, 453-458.

Wang, X.T. (1996a). Domain-specific rationality in human choices: violations of utility axioms and social contexts. Cognition, 60, 31-63.

Wang, X.T. (1996b). Framing effects: Dynamics and task domains. Organizational Behavior and human decisions processes, 68 , 145-157. 